\title{
Seroprevalences of Classical Swine Fever Virus and Porcine Reproductive and Respiratory Syndrome Virus in Pigs in Hunan Province, China
}

Haoyang Yu

Hunan Agricultural University

Luhua Zhang

Hunan Agricultural University

Yunfeng Cai

Hunan Agricultural University

Tao Peng

Hunan Agricultural University

Lei Liu

Hunan Agricultural University

Naidong Wang

Hunan Agricultural University

Guiping Wang

Hunan Agricultural University

Zhibang Deng

Hunan Agricultural University

Yang Zhan ( $\square$ yangzhan@hunau.edu.cn )

Hunan Agricultural University https://orcid.org/0000-0002-1881-6249

\section{Research article}

Keywords: Classical swine fever virus (CSFV), Porcine reproductive and respiratory syndrome virus (PRRSV), Pig, Seroprevalence, Antibody

Posted Date: August 7th, 2020

DOI: https://doi.org/10.21203/rs.3.rs-41387/v1

License: (c) (1) This work is licensed under a Creative Commons Attribution 4.0 International License.

Read Full License 


\section{Abstract}

Background: Several infectious diseases including classical swine fever (CSF) and porcine reproductive and respiratory syndrome (PRRS) are responsible for major economic losses and represent a threat to the swine industry worldwide. Hunan is a province in south-central China, serological statistics will have certain effect on local breeding industry.

The present study investigated the seroprevalences of classical swine fever virus (CSFV) and porcine reproductive and respiratory syndrome virus (PRRSV) in pigs from eight cities of Hunan province from 2017 to 2019. The samples were divided into two groups based on whether the hosts were immunized or not, and analysed by enzyme-linked immunosorbent assay (ELISA).

Results: The seropositivity of CSFV and PRRSV between different parts of Hunan province in China was statistically significant. In unvaccinated group, our findings showed CSFV antibody in piglets' sera decreases gradually with increase of piglets' age whereas PRRSV-specific antibodies may appear in various growth stages. In immunized group, the average seropositive rate of CSFV antibody was $82.2 \%$, while the rate of PRRSV antibody was relatively higher (84.4\%).

Conclusions: Results from this study suggested that a serological investigation on these two major infectious diseases (CSF and PRRS) among various growth stages of pigs will provide a basis for further development of more effective preventions and control measures.

\section{Background}

Classical swine fever (CSF) is listed as a major notification disease by the World Organization for Animal Health (OIE) and listed as a Class A animal epidemic disease in China [1]. Classical swine fever virus (CSFV) is a small RNA virus with envelope in the Flaviviridae family, and the genome is approximately $12.3 \mathrm{~kb}$ in length [2]. Importantly, CSF is a highly infectious disease that occurs in both piglets and adult pigs by contact, and causes persistent infections as CSFV can evade the host immune surveillance [3]. CSF has been endemic in China since the early 20th century, and the prevention and control of this disease mainly depends on prophylactic vaccination, such as the Chinese lapinized vaccine [4]. However, compared to the classic strain, some newly emerging CSFV isolates could also be epidemic in many immunized pig farms or sporadically isolated from immunized pig farms, and showed distinct virulence and clinical manifestations $[5,6]$.

Porcine reproductive and respiratory syndrome virus (PRRSV), an enveloped and single positive-stranded RNA virus, belongs to a member of the family Arteriviridae [7]. It has been determined the key etiologic agent responsible for porcine reproductive and respiratory syndrome (PRRS) since the early 1990s [8]. PRRSV has been epidemic more than 20 years in China, and administration of PRRSV vaccines has provided an efficient protection from the virus infection by reducing clinical signs, decreasing viremia and shortening duration of viral shedding [9]. To date, the existing commercial vaccines, mostly known as modified live attenuated viruses, provide the greatest levels of protection [10]. However, the attenuated 
vaccines fall short in a variety of areas [11]. Modified live attenuated viruses may cause chronic or persistent infections and have the potential to revert to virulence, bringing new challenges to PRRSV prevention and control in Chinal

In order to accelerate the eradication of CSFV and PRRSV, the serology of both virus infections in the context of the current immunization programs should be surveyed and vaccine administrations also need to be evaluated to efficiently manage these virus-associated diseases on farms.

In this study, CSFV and PRRSV antibodies were tested in serum samples from immunized and unvaccinated pigs in different parts of Hunan province of China from 2017 to 2019, to evaluate vaccine efficacy and serological prevalence of both viruses.

\section{Results}

\section{Seroprevalence-specific rates of unvaccinated pigs.}

A total of 129 unvaccinated pigs from eight regions were sampled for testing CSFV antibody, and $77.5 \%$ (100/129) were positive for CSFV (Table 1). Furthermore, $0(0 / 10)$ and $100 \%(4 / 4)$ in the regions of Chenzhou and Yueyang were the positive rates for CSFV as a minimum and maximum, respectively, and the seropositivity of CSFV between the sample locations $(n=8)$ was statistically significant by using chisquare test $\left(X^{2}=43.648, \mathrm{df}=7, p<0.001\right)$. The results showed that no CSFV infection on fattening pigs $(0 / 10)$, whereas all the positive CSFV samples were from the piglets $(100 / 100)$. It is worth to note that CSFV antibody in piglets decreased gradually with increase of piglets' age (Fig. 2A). 
Table 1

Seroprevalence-specific rates of CSFV/PRRSV in pigs by sampling location

\begin{tabular}{|lllll|}
\hline Category & \multicolumn{2}{l}{ CSFV antibodies test } & \multicolumn{3}{l|}{ PRRSV antibodies test } \\
\cline { 2 - 5 } & $\begin{array}{l}\text { Positive no. / No. of } \\
\text { unvaccinated pigs } \\
\text { sampled (positive } \\
\text { rate) }\end{array}$ & $\begin{array}{l}\text { Positive no. / No. } \\
\text { of immune pigs } \\
\text { sampled (positive } \\
\text { rate) }\end{array}$ & $\begin{array}{l}\text { Positive no. / No. of } \\
\text { unvaccinated pigs } \\
\text { sampled (positive } \\
\text { rate) }\end{array}$ & $\begin{array}{l}\text { Positive no. / No. } \\
\text { of immune pigs } \\
\text { sampled (positive } \\
\text { rate) }\end{array}$ \\
\hline Changsha & $2 / 4(50 \%)$ & $93 / 122(76.2 \%)$ & $/$ & $111 / 126(88.1 \%)$ \\
\hline Hengyang & $8 / 10(80 \%)$ & $128 / 152(84.2 \%)$ & $76 / 151(50.3 \%)$ & $17 / 23(73.9 \%)$ \\
\hline Shaoyang & $20 / 27(74.1 \%)$ & $112 / 139(80.6 \%)$ & $48 / 93(51.6 \%)$ & $68 / 73(93.2)$ \\
\hline Yueyang & $4 / 4(100 \%)$ & $259 / 299(86.6 \%)$ & $6 / 9(66.7 \%)$ & $228 / 270(84.4 \%)$ \\
\hline Changde & $54 / 60(90 \%)$ & $364 / 449(81.1 \%)$ & $116 / 310(37.4 \%)$ & $255 / 320(79.7 \%)$ \\
\hline Yiyang & $4 / 5(80 \%)$ & $33 / 39(84.6 \%)$ & $33 / 35(94.3 \%)$ & $23 / 24(95.8 \%)$ \\
\hline Chenzhou & $8 / 9(88.9 \%)$ & $65 / 88(73.9 \%)$ & $27 / 38(71.1 \%)$ & $53 / 59(89.8 \%)$ \\
\hline Yongzhou & $0 / 10(0)$ & $31 / 32(96.9 \%)$ & $/$ & $38 / 40(95 \%)$ \\
\hline Total & $100 / 129(77.5 \%)$ & $1085 / 1320$ & $306 / 636(48.1 \%)$ & $793 / 935(84.8 \%)$ \\
\hline
\end{tabular}

For detecting PRRSV antibody, 636 serum samples from pigs without vaccination against PRRSV were detected by ELISA, and the average positive rate of PRRSV-specific antibody was $48.1 \%$ (Table 1). PRRSV appeared in the six regions of Hunan province, and the highest positive rate was $94.3 \%$ in Yiyang, whereas the lowest was $37.4 \%$ in Changde (Table 1). The seropositivity of PRRSV between the sample locations $(n=6)$ was also statistically significant by using chi-square test $\left(\chi^{2}=54.095, d f=5, p<0.001\right)$. Moreover, the highest positive rate in different growth stages was $77.8 \%$ for boars, and the lowest was $18.9 \%$ for fattening pigs (Fig. 2B). Unlike CSFV, PRRSV antibody in piglets first increased from one week old to two weeks old, and then decreased gradually with increase of piglets' age (Fig. 2B).

\section{Seroprevalence-specific rates of immunized pigs.}

Vaccination efficacies against CSFV has been studied in Hunan province, and the positive rates of CSFV antibody was $82.2 \%$ by detecting 1320 serum samples in Hunan province (Table 1). By analysis of regional differences, the vaccination efficacy in Yongzhou was best with the sera-positive rate is $96.9 \%$, but the positive rates of four regions (Changsha, Shaoyang, Changde, and Chenzhou) were below the average (Table 1). Further statistical analysis, the seropositivity of CSFV between the sample locations ( $\mathrm{n}$ $=8$ ) was statistically significant by using chi-square test $\left(\chi^{2}=17.074, \mathrm{df}=7, p=0.017\right)$. The vaccination efficacy was not uniform at different growth stages, the positive rate from high to low was boars (92.6\%), sows $(90 \%)$, piglets $(75.9 \%)$, fattening pigs $(69.3 \%)$, and nursery pigs $(50.3 \%)$ (Table 2 ). 
Table 2

Positive rate of CSFV antibody in immune pigs of different growth stage

\begin{tabular}{|llll|}
\hline Growth stage & No. of tested samples & No. of positive samples & Positive rate \\
\hline Piglets & 54 & 41 & $75.9 \%$ \\
\hline Nursery pigs & 145 & 73 & $50.3 \%$ \\
\hline Fattening pigs & 192 & 133 & $69.3 \%$ \\
\hline Sows & 835 & 751 & $90 \%$ \\
\hline Boars & 94 & 87 & $92.6 \%$ \\
\hline Total & 1320 & 1085 & $82.2 \%$ \\
\hline
\end{tabular}

Vaccination efficacies against PRRSV were also studied in Hunan province, the positive rate of PRRSV antibody in sera was $84.4 \%$ by detecting 935 serum samples (Table 1). About the regional difference, the highest positive rate was $95.8 \%$ in Yiyang with the best vaccination efficacy, and the lowest was $74 \%$ in Hengyang (Table 1). The seropositivity of PRRSV between the sample locations $(n=8)$ was also significantly different using chi-square test $\left(X^{2}=20.309, d f=7, p=0.005\right)$. Among various growth stages, the positive rate of sows was highest (88.1\%) implying that the vaccination efficacy reached a high level, the lowest rate was $28.6 \%$ in piglets, which indicated a poor vaccination efficacy (Table 3 ).

Table 3

Positive rate of PRRSV antibody in immune pigs of different growth stage

\begin{tabular}{|llll|}
\hline Growth stage & No. of tested samples & No. of positive samples & Positive rate \\
\hline Piglets & 14 & 4 & $28.6 \%$ \\
\hline Nursery pigs & 48 & 29 & $60.4 \%$ \\
\hline Fattening pigs & 68 & 56 & $82.4 \%$ \\
\hline Sows & 720 & 634 & $88.1 \%$ \\
\hline Boars & 85 & 70 & $82.4 \%$ \\
\hline Total & 935 & 793 & $84.8 \%$ \\
\hline
\end{tabular}

\section{Discussion}

The transportations of people and pigs are apparently different now from that in the past, and this has led to an increasing concern about viruses, such as CSFV or PRRSV, spreading to the pathogen-free areas, leading not only to animal infections, but also to economic loss in pig industry [12]. Hunan is the thirdlargest swine-raising province in China, and serological statistics of CSFV and PRRSV antibodies in Hunan province have certain effect on the whole breeding industry. 
The prevalence of $77.5 \%$ is a serological indication that CSF may exist in this study area without of using CSFV vaccine. However, Chen at al. similarly reported that only 4 pigs were solely infected with CSFV among 159 diseased pigs in China from 2016 to 2018 [13]. Thus, our prevalence (100/129, 77.5\%) tested by ELISA is significantly higher than the reported positive percentage of CSFV $(4 / 159,2.52 \%)$ detected by real-time PCR. It is possible that all the positive CSFV samples were from the unvaccinated piglets while their mothers were immunized against CSFV, therefore the serological level of CSFV antibody in the nonvaccinated pigs comes from the maternally derived antibodies (MDA), and it decreases with the age of piglets. It is worth noting that CSFV vaccination should be administered when residual MDA are minimal and before pigs become naturally infected, avoiding potential interference from MDA at the time of vaccination [14].

What distinguishes CSFV from PRRSV? The answer is that PRRSV antibody in the unvaccinated piglets (their mothers were immunized against PRRSV) increases first and then reduces with the ages. Further, more unvaccinated pigs from different growth stages (nursery pigs, sows, and boars) were used to detect PRRSV antibody which can reflect the prevalence of PRRSV in corresponding region. The presence of the virus in each growth stage (except piglets) presented obvious different positive rates: $71.7 \%$ for nursery pigs, $18.9 \%$ for fattening pigs, $53.4 \%$ for sows, and $77.8 \%$ for boars. High positive of boars in this study, may explain that PRRSV was widespread in pig farms.

Vaccination efficacies against CSFV or PRRSV have also been studied in Hunan province. Only the serapositive rate of CSFV antibody in Yongzhou was above $90 \%$, showing an ideal vaccination efficacy, but other areas in Hunan province need enhance the efficacy of vaccines. On the different growth stage of CSFV-immune pigs, sows and boars achieved a high level of vaccination efficacy, while others (piglets, fattening pigs, and nursery pigs) were not satisfactory yet. It is considered to be the ideal immune effect when the positive rate of PRRSV antibody in immune pig farms is above $70 \%$. Our study showed that most PRRSV-immune pigs from areas in Hunan province under investigation got the antibody (84.4\%). However, the positive rate of piglets was $28.6 \%$, less than $70 \%$. This low percentage imply that the efficacy of vaccination in piglets could be affected by the presence of MDA.

\section{Conclusion}

This study has determined the serological statistics of CSFV and PRRSV antibodies from immunized and unvaccinated pigs in Hunan province of China from 2017 to 2019. Continuous monitoring of these viral antibodies among pigs in China is hereby recommended as well as improved immune procedure with intensified awareness campaign.

\section{Methods}

\section{Study locations and design}


Hunan province is located in the south-central region of China, a farming zone known for high pig production. The sampling area is consisted of 8 herds and the herds were located in the north eastern (Changsha and Yueyang), northern (Changde and Yiyang), southern eastern (Chenzhou), and southern (Hengyang, Shaoyang, Yongzhou) parts of Hunan province (Fig. 1). The number of pigs to be sampled per herd was based on the availability of pigs and willingness of the farmers to allow for their pigs to be sampled. Furthermore, Face-to-face interviews were carried out with farmers ( $>18$ years old) who were mostly involved in swine breeding and management, and filled the registration form covered information of samples, such as the growth stage, age, and the immunity condition of pigs (Additional file 1).

For detecting CSFV antibodies, a total of 1449 blood samples from pigs corresponding to nonvaccination $(n=129)$ and vaccination $(n=1320)$ of CSFV, different size herds sampled during years 2017-2019 were used (Fig. 1). Also, totally 1571 swine blood samples relevant to non-vaccination or vaccination of PRRSV, from the same herds were collected for testing PRRSV antibodies (Fig. 1).

Three millilitres of blood sample was collected from each pig by using a $5 \mathrm{ml}$ syringe and corresponding needle. The blood was then gently transferred into a $5 \mathrm{ml}$ sterile tube, labeled appropriately and standing overnight. Then, the blood was centrifuged at $3000 \times \mathrm{g}$ for $10 \mathrm{~min}$ to separate sera from clotted blood. The sera were then decanted into an appropriately labeled $2 \mathrm{ml}$ plastic serum storage tube, and stored at $-20^{\circ} \mathrm{C}$ until used.

\section{Laboratory analysis}

Two commercial kits: IDEXX PRRS X3 Ab Test (indirect for PRRS) and IDEXX CSFV Ab Test (E2 competitive for CSF) from IDEXX Laboratories, Inc. (Westbrook, ME, USA) were used to test for the presences of PRRSV and CSFV antibodies in sera, respectively. The manufacturer's instructions were strictly adhered to and results were read and interpreted using an ELISA reader at a wavelength of 450 or $650 \mathrm{~nm}$. For PRRSV, samples are considered positive if the S/P ratio is $\geq 0.4$ and negative if the ratio < 0.4 , where $\mathrm{S} / \mathrm{P}=\left(\right.$ sample $_{\mathrm{OD} 650}$ - negative control $\left.{ }_{\mathrm{OD} 650}\right) /\left(\right.$ positive control $_{\mathrm{OD} 650}$ - negative control $\left.{ }_{\mathrm{OD} 650}\right)$.

For CSFV, samples are considered positive if the Blocking $\%$ is $\geq 0.4$ and negative if $\leq 0.3$, where Blocking $\%=\left(\right.$ negative control $\mathrm{OD} 450_{0}-$ sample $\left._{\mathrm{OD} 450}\right) /$ negative control $\mathrm{OD} 450_{0} \times 100$.

\section{Data analysis}

All statistical analyses were performed using SPSS software (Version 20, Chicago, USA).

\section{Abbreviations}

CSFV: Classical swine fever virus; PRRSV: Porcine reproductive and respiratory syndrome virus; ELISA: Enzyme-linked immunosorbent assay; OIE: World Organization for Animal Health

\section{Declarations}


Acknowledgements

The authors thank all farmers from pig farms that participated in this study.

\section{Authors' contributions}

Conceived and designed the study: ZY, DZB, WND, and WGP. Performed the experiments and analyzed data: YHY, ZLH, CYF, and PT. Wrote the paper: YHY, ZLH, and ZY. Revised the manuscript: LL and WND. All authors read and approved the final manuscript.

\section{Funding}

This work was supported by Hunan Provincial Key Laboratory of Protein Engineering in Animal Vaccines (Grants No. 2017TP1014) for collecting blood samples. This work was also funded by Hunan Education Department's Science \& Research Project (Grant No. 18B092) for detecting CSFV/PRRSV antibodies in sera. The funds did not involve in the design of the study, analysis and interpretation of data and in writing the manuscript.

\section{Availability of data and materials}

All datasets supporting our findings are available from the corresponding author on reasonable request.

\section{Ethics approval and consent to participate}

All experiments were approved and reviewed by the Ethics Committee for Biomedical Research of Hunan Agricultural University, Changsha, China following the national legislations regarding animal's welfare. Furthermore, the owners of the pig farms were inquired for getting verbal permission to conduct this study.

\section{Consent for publication}

Not applicable.

\section{Competing interests}

The authors declared that they have no conflicts of interest to this work.

\section{References}

1. Zhou B. Classical Swine Fever in China-An Update Minireview. Front Vet Sci. 2019;6:187.

2. Brown VR, Bevins SN. A Review of Classical Swine Fever Virus and Routes of Introduction into the United States and the Potential for Virus Establishment. Front Vet Sci. 2018;5:31.

3. Goraya MU, Ziaghum F, Chen S, Raza A, Chen Y, Chi X. Role of innate immunity in pathophysiology of classical swine fever virus infection. Microbial pathogenesis. 2018;119:248-254. 
4. Luo YZ, Li S, Sun Y, Qiu HJ. Classical swine fever in China: A minireview. Veterinary microbiology. 2014;172:1-6.

5. Zhang HL, Leng CL, Tian ZJ, Liu CX, Chen JZ, Bai Y, et al. Complete genomic characteristics and pathogenic analysis of the newly emerged classical swine fever virus in China. BMC veterinary research. 2018;14:204.

6. Gong WJ, Li JH, Wang ZB, Sun JM, Mi SJ, Lu ZJ, et al. Virulence evaluation of classical swine fever virus subgenotype 2.1 and 2.2 isolates circulating in China. Veterinary microbiology. 2019;232:114120.

7. Han MY, Yoo DW. Engineering the PRRS virus genome: Updates and perspectives. Veterinary microbiology. 2014;174:279-295.

8. Montaner-Tarbes S, del Portillo HA, Montoya M, Fraile L. Key Gaps in the Knowledge of the Porcine Respiratory Reproductive Syndrome Virus (PRRSV). Front Vet Sci. 2019;6:38.

9. Guo ZH, Chen XX, Li R, Qiao SL, Zhang GP. The prevalent status and genetic diversity of porcine reproductive and respiratory syndrome virus in China: a molecular epidemiological perspective. Virology journal. 2018;15:2.

10. Madapong A, Saeng-Chuto K, Chaikhumwang P, Tantituvanont A, Saardrak K, Pedrazuela Sanz R, et al. Immune response and protective efficacy of intramuscular and intradermal vaccination with porcine reproductive and respiratory syndrome virus 1 (PRRSV-1) modified live vaccine against highly pathogenic PRRSV-2 (HP-PRRSV-2) challenge, either alone or in combination with of PRRSV-1. Veterinary microbiology. 2020;244:108655.

11. Stoian A, Rowland R. Challenges for Porcine Reproductive and Respiratory Syndrome (PRRS) Vaccine Design: Reviewing Virus Glycoprotein Interactions with CD163 and Targets of Virus Neutralization. Veterinary Sciences. 2019;6:9.

12. VanderWaal K, Deen J. Global trends in infectious diseases of swine. Proceedings of the National Academy of Sciences of the United States of America. 2018;115:11495-11500.

13. Chen N, Huang Y, Ye M, Li S, Xiao Y, Cui B, et al. Co-infection status of classical swine fever virus (CSFV), porcine reproductive and respiratory syndrome virus (PRRSV) and porcine circoviruses (PCV2 and PCV3) in eight regions of China from 2016 to 2018. Infection, genetics and evolution. 2019;68:127-135.

14. Farsang A, Lévai R, Barna T, Fábián K, Blome S, Belák K, et al. Pre-registration efficacy study of a novel marker vaccine against classical swine fever on maternally derived antibody positive (MDA+) target animals. Biologicals. 2017;45:85-92.

\section{Figures}




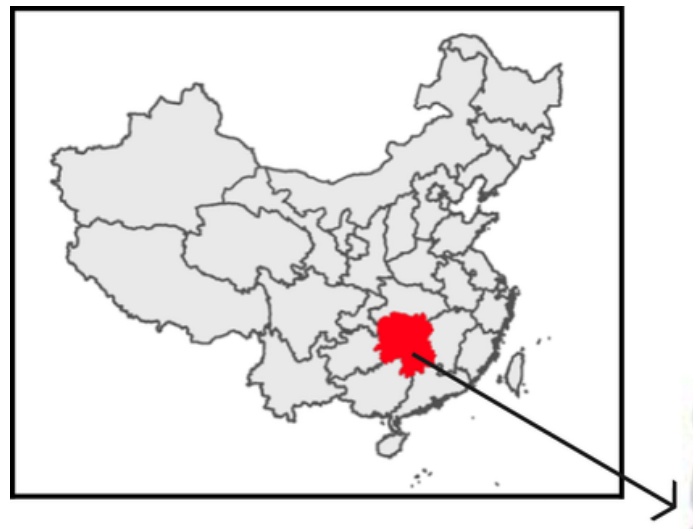

$\triangle \quad$ CSFV unvaccinated pigs

$\square \quad$ PRRSV unvaccinated pigs

A CSFV-immune pigs

PRRSV-immune pigs

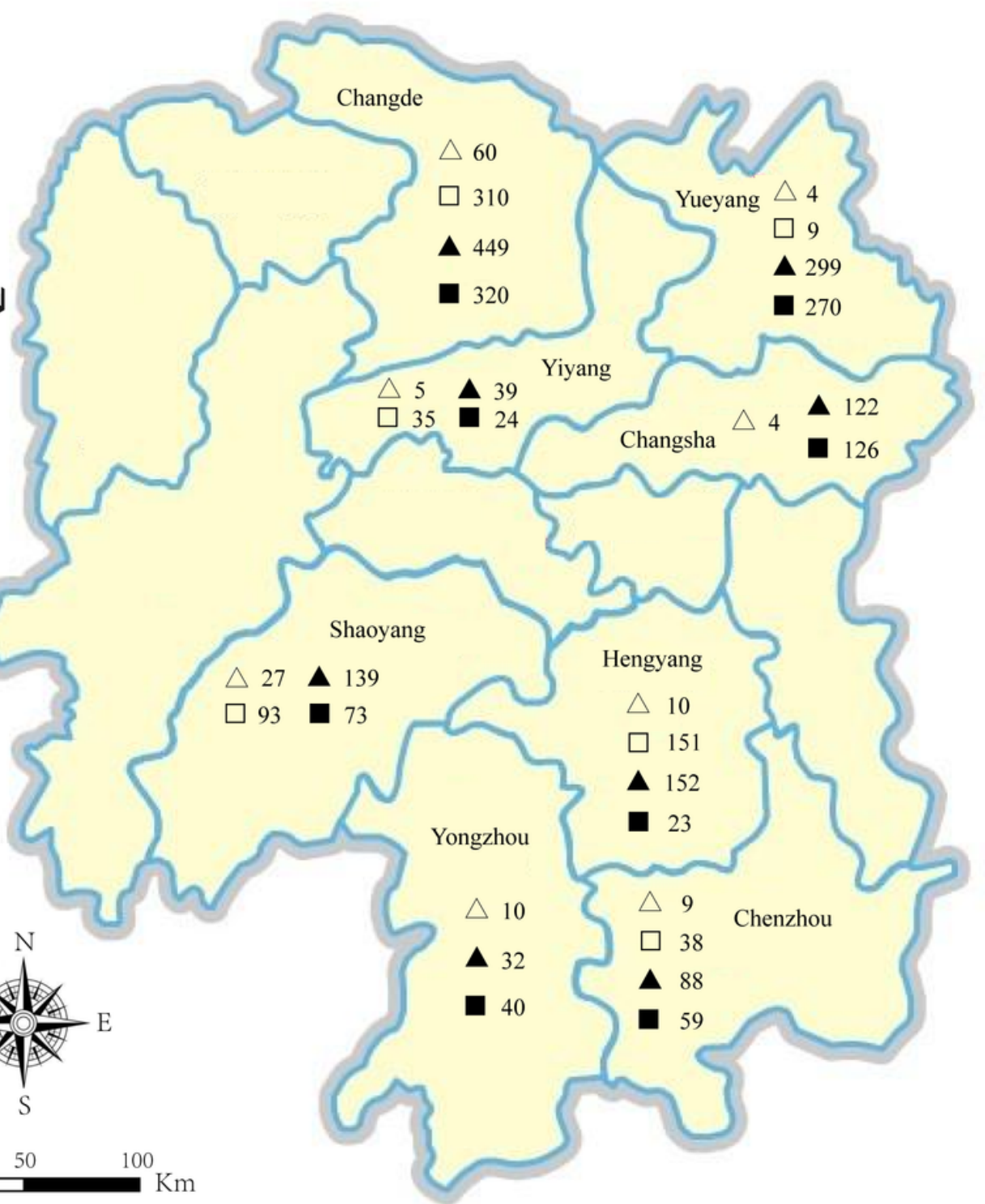

Figure 1

Geographic distribution of the swine herds in this study Map (displayed enlarged area), with location of Hunan province in China highlighted in red and the herds in different parts of the Hunan were labeled: Changsha, Hengyang, Shaoyang, Yueyang, Changde, Yiyang, Chenzhou, and Yongzhou. Hollow triangle $(\triangle)$ or hollow square $(\square)$ represents pigs without CSFV (A) /PRRSV(B) vaccination, respectively. Filled triangle $(\boldsymbol{\Delta})$ or filled square $(\boldsymbol{\square})$ represents CSFV (A) /PRRSV(B)-immune swine samples. The number of samples of each group is shown with the corresponding shape. Acknowledge: The geographical maps used in this figure is from the network material (website: http://www.51yuansu.com/), and the geographical maps is free of copyright and can be used for free. Note: The designations employed and the presentation of the material on this map do not imply the expression of any opinion whatsoever on 
the part of Research Square concerning the legal status of any country, territory, city or area or of its authorities, or concerning the delimitation of its frontiers or boundaries. This map has been provided by the authors.

A

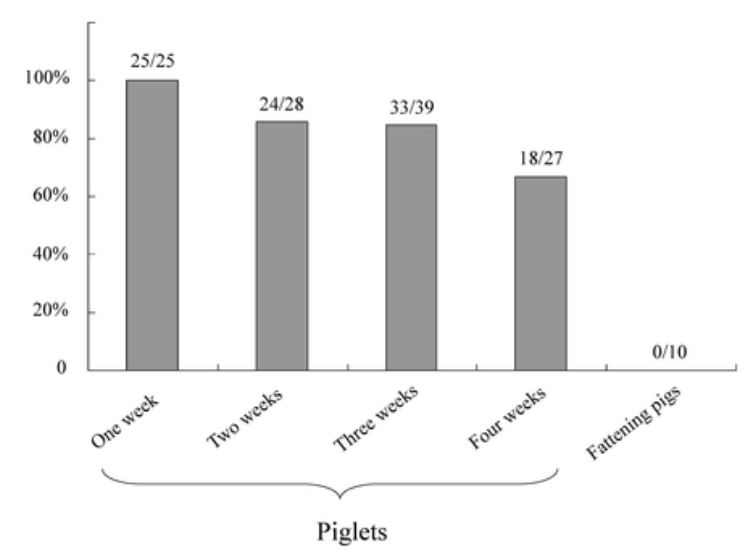

B

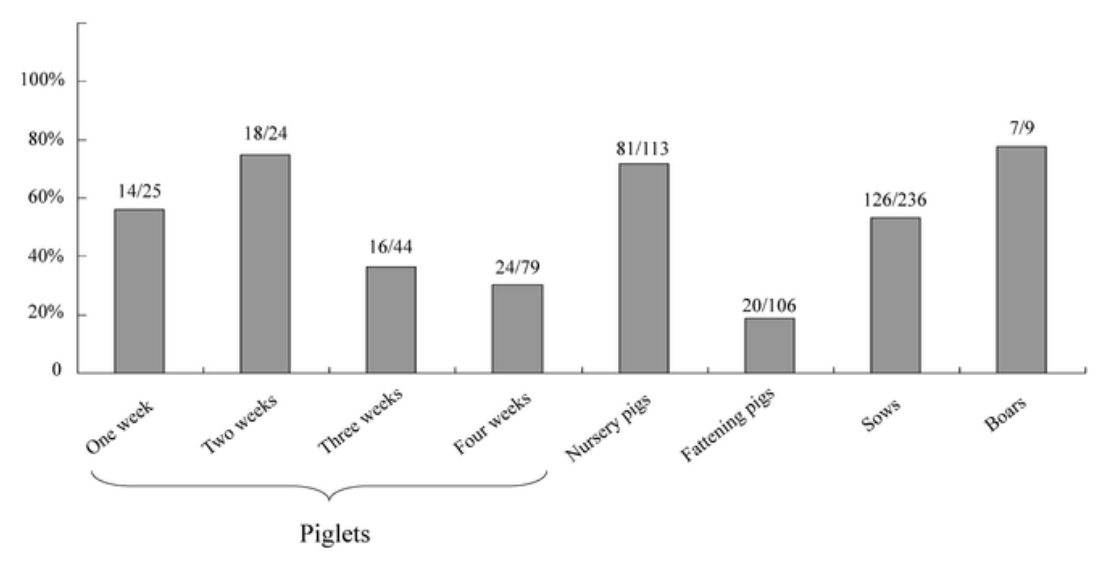

Figure 2

Positive rate of CSFV/PRRSV antibody in unvaccinated pigs of different growth stage Note: The ratio above each column is the number of positive samples divided by the number of test samples.

\section{Supplementary Files}

This is a list of supplementary files associated with this preprint. Click to download.

- Additionalfile2AuthorChecklist.pdf

- Additionalfile1Registrationformofdetectedswinedata.doc 